\title{
OPPIS Upgrade for 2003 Polarized Run in RHIC $^{\text {a }}$
}

\author{
A. Zelenski", J. Alessi", B. Briscoe ${ }^{*}$, A. Kponou', S. Kokhanovski", \\ V. Klenov ${ }^{\dagger}$, V. LoDestro*, J. Ritter ${ }^{*}$, V. Zubets ${ }^{\dagger}$ \\ *Brookhaven National Laboratory, USA \\ ${ }^{\dagger}$ INR, Moscow, Russia
}

\begin{abstract}
The polarization dilution by molecular ions which are produced in the ECR primary proton source is discussed. The molecular component can be reduced to about $5 \%$ by ECR source-operation optimization. It is further suppressed by optimization of the extraction electrode optics and by the decelerating einzel lens in the $35 \mathrm{keV}$ LEBT line. As a result, the proton polarization of the accelerated beam was increased to over $80 \%$, as measured in the $200 \mathrm{MeV}$ proton-deuterium polarimeter. The OPPIS upgrade for $62 / 3 \mathrm{~Hz}$ repetition rate operation is also discussed.
\end{abstract}

\section{INTRODUCTION}

The RHIC OPPIS produces routinely $0.5-1.0 \mathrm{~mA}$ (maximum $1.5 \mathrm{~mA}$ ) current in a $400 \mu$ s pulse duration. Polarized $\mathrm{H}^{-}$ions are produced in the OPPIS at $35 \mathrm{keV}$ beam energy. The beam is accelerated to $200 \mathrm{MeV}$ with an RFQ and a linac for charge exchange strip-injection into the Booster. About $50 \%$ of the OPPIS beam intensity can be accelerated to $200 \mathrm{MeV}$. The $400 \mu \mathrm{s} \mathrm{H}$ ion pulse is captured in a single Booster bunch which contains about $4 \cdot 10^{11}$ polarized protons. The single bunch is accelerated in the Booster to $1.5 \mathrm{GeV}$ kinetic energy and then transferred to the AGS, where it is accelerated to $25 \mathrm{GeV}$ for injection to RHIC.

The OPPIS' initial longitudinal polarization is converted to the transverse direction while the beam passes two bending magnets.The second $47.4^{\circ}$ bending magnet switches linac injection between polarized and unpolarized high intensity (up to 100 $\mathrm{mA}) \mathrm{H}^{-}$ion beam. The magnet is pulsed and either beam can be accelerated pulse-topulse in the same RFQ. A pulsed focusing solenoid in front of the RFQ is tuned for optimal transmission for either beam. It rotates the polarization direction for about $420^{\circ}$, but still keeps it in the transverse plane, and a final polarization alignment to the vertical direction can be adjusted by a spin-rotator solenoid in the $750 \mathrm{keV}$ beam transport line before injection to the linac [2]. The AGS cycle for polarized beam operation is 3 seconds. The OPPIS operates at $1 \mathrm{~Hz}$ repetition rate and additional source pulses were directed to the $200 \mathrm{MeV}$ p-Carbon polarimeter for continuous polarization monitoring by switching of another pulsed bending magnet in the high- 
energy beam transport line. The spin-rotator tuning is done using vertical polarimeter arms.

\section{OPPIS PERFORMANCE}

Recent source component upgrades have significantly improved the OPPIS performance and reliability. The OPPIS polarization technique is described elsewhere $[3,4]$. The schematic OPPIS layout is presented in Fig.1.

The ECR-primary proton source upgrade: The ECR operation in a pulsed mode was studied at $1-7 \mathrm{~Hz}$ repetition rate and 5-100 ms pulse duration. A significant reduction in the optimal hydrogen feeding flow was observed, which might be explained by gas adsorption on the quartz tube, then desorption at the beginning of the $R F$ pulse. This produces additional gas contribution sufficient to maintain an optimal density for about 5-10 msec at the beginning of the pulse. Pulsed ECR operation reduces gas load to the vacuum system and heat load to the ECR and Rb cell. In dc operation an oxygen gas admixture is required to optimize the ECR-source proton production and proton to molecular $\mathrm{H}_{2}{ }^{+}$ions dissociation ratio. In the pulsed operation sufficient amount of oxygen is supplied to the discharge from residual gas.

Advantages of the pulsed operation were not fully used in the RHIC run, because the heat load difference between pulsed and dc operation eventually caused leaks in a quartz to copper cavity seal. In the new ECR cavity silicone O-rings (silicone has lower RF-power absorption than other rubber-like materials) are used instead of a teflon-indium seal. The silicone O-ring exposure to $29 \mathrm{GHz}$ microwave power is reduced by the seal design. The quartz tube air-cooling was improved to prevent seal overheating through the contact with the hot quartz tube.

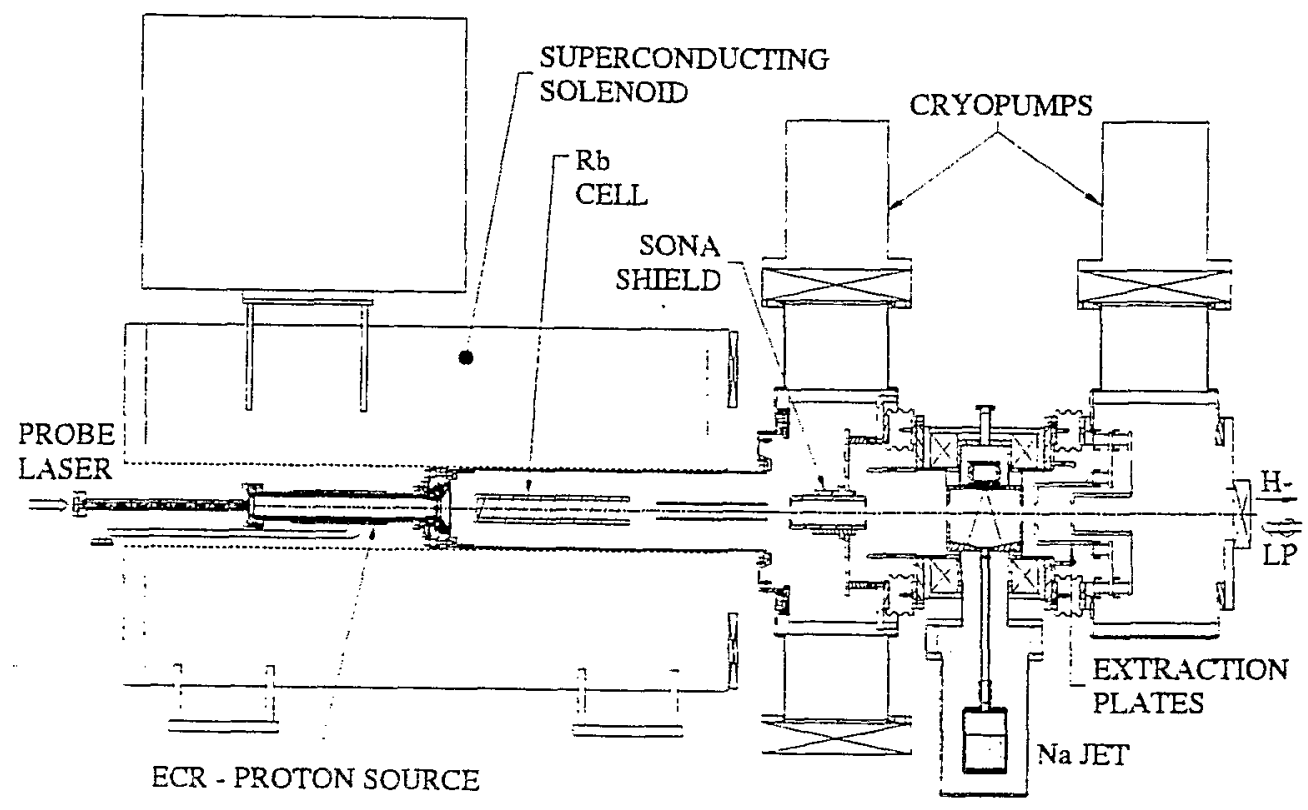

FIGURE 1.: The RHIC OPPIS layout. 


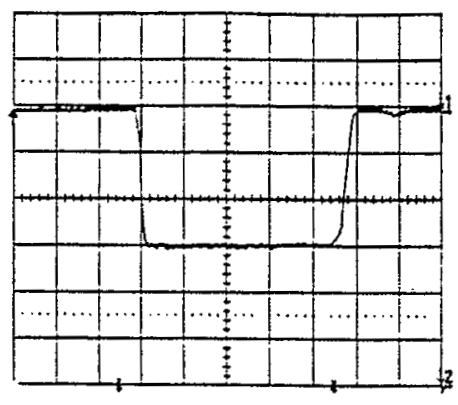

FIGURE 2. The polarized H- ion beam current pulse in a $35 \mathrm{keV}$ LEBT Faradey cup.

Vertical scale- $500 \mu \mathrm{A} / \mathrm{div}$, horizontal-100 $\mu \mathrm{sec} / \mathrm{div}$.

The silicone O-rings provide the flexibility to compensate the difference in thermal expansion between quartz and copper, which allows frequent switching between dc and pulsed modes for optimal source operation. The new ECR-cavity length was also increased to match the magnetic field shape in the ECR-discharge region. As a result the maximum polarized $\mathrm{H}$ ion current was increased to $1.5 \mathrm{~mA}$ (see Fig. 2).

Laser system upgrade: The laser pulse duration was extended to $450 \mu$ for optical pumping during $400 \mu \mathrm{s} \mathrm{H}^{-}$current pulse. On-line rubidium polarization measurements were implemented, by probe laser linear polarization rotation measurements (Faraday rotation polarimeter). These measurements give a reliable polarization readout for confirmation of the spin sequence pattern, which is injected to RHIC. Recently, the laser was successfully tested at a $7 \mathrm{~Hz}$ OPPIS repetition rate.

\section{POLARIZATION STUDIES}

Over $85 \%$ polarization was obtained during the final RHIC OPPIS tests at TRIUMF where only electrostatic beam optics were used [4]. A 70-72\% polarization was measured in the first year 2000 run at $200 \mathrm{MeV}$ in the p-carbon polarimeter. at a reduced intensity of less than $10 \mu \mathrm{A}$. (The polarimeter was designed for the low current atomic beam source and detectors where greatly overloaded at $200 \mu \mathrm{A}$ current.) The polarimeter was upgraded for high current operation by extending target to detector distance from 70 to $250 \mathrm{~cm}$ and additional collimator installation to suppress particles scattered from the target holder. Polarization measurements at $180 \mu \mathrm{A}$ beam intensity were obtained during spin-rotator tuning. Still there is some saturation. and measured polarization is higher at $120 \mathrm{uA}$. The p-carbon polarimeter is inclusive and relied on the calibration measurements, which were done 10 years ago under different conditions. The absolute polarization is an important reference point for polarization loss measurements in Booster and AGS and for evaluation of the OPPIS development status. An upgrade was suggested an upgrade to a polarimeter using p-deutron elastic scattering, where the analyzing power is precisely known at $200 \mathrm{MeV} / 5 /$. Four additional detector arms for the proton-deutron coincidence measurements from $C D_{2}$. 
target (deuteriated polyethylene) were installed in the summer of 2001. During the November 2001-January 2002 run, p-C and p-D scattering data were accumulated and the old analyzing power for $12^{\circ}$ degree inclusive $\mathrm{pC}$ scattering of a 0.62 was closely reproduced [6]. Therefore the $70-75 \%$ polarization at $200 \mathrm{MeV}$ was real and lower than expected.

\section{POLARIZATION DILUTION BY $\mathrm{H}_{2}^{+}$MOLECULAR IONS}

There exists a molecular $\mathrm{H}_{2}{ }^{+}$ion component in any plasma ion source. In the OPPIS, molecular ions after dissociation will appear as $\mathrm{H}^{-}$ions with the half of the primary beam energy. The polarization of this beam might be different from the main beam (measurement in Lamb-shift polarimeter gives about half the polarization for this molecular component). This component was observed at the TRIUMF OPPIS, but it was efficiently suppressed by electrostatic lenses in the $3 \mathrm{keV} \mathrm{LEBT}$. In the RHIC OPPIS the $\mathrm{H}^{-}$beam is accelerated for $32 \mathrm{keV}$ immediately after ionization, producing $35 \mathrm{keV}$ main beam and $33.5 \mathrm{keV}$ beam from molecular ion admixture. These beams are not well separated in the LEBT, and the molecular component is responsible for polarization dilution. At lower acceleration energy these beams are separated, and the half energy component was directly observed (see Fig.3).

A value of molecular component of about $10-40 \%$ was measured under different ECR conditions. Since every $\mathrm{H}_{2}{ }^{+}$ion is dissociated to two half energy atoms, it means 5-20\% molecular component out of the ECR-source. The molecular component increases at higher ECR extraction voltage. At $4.0 \mathrm{keV}$ its value is about $40 \%$. The $\mathrm{H}^{-}$ yield drops at atomic beam energy above $3.0 \mathrm{keV}$, but for half energy beam of a 1.5 $2.0 \mathrm{keV}$ the yield is at maximum value. It explains an increase of molecular component up to $40 \%$ at $4.0 \mathrm{keV}$ ECR extraction energy, and correspondent polarization decrease, which was also observed in polarization measurements.

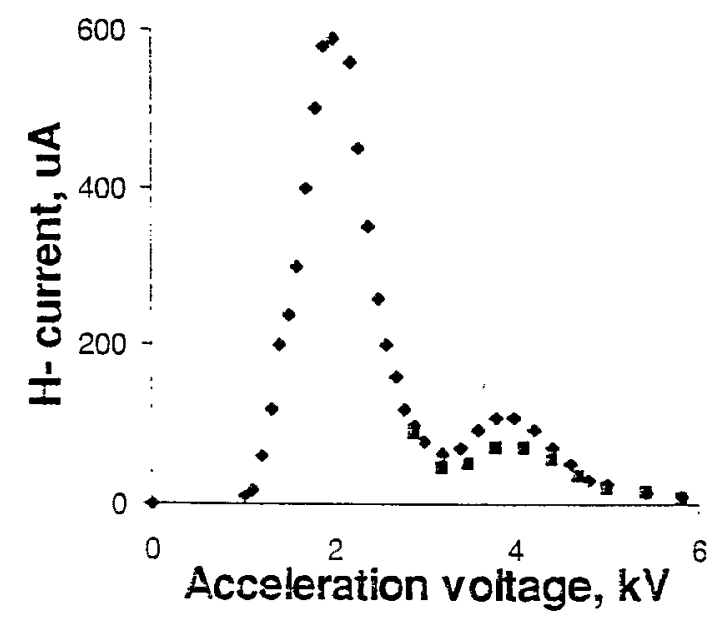

FIGURE 3. Molecular $\mathrm{H}_{2}^{-}$beam component is appeared as a second bump shifted to about a half primary ECR proton energy at a fixed bending magnet serting. Diamonds-dc operation: squares-pulsed. 


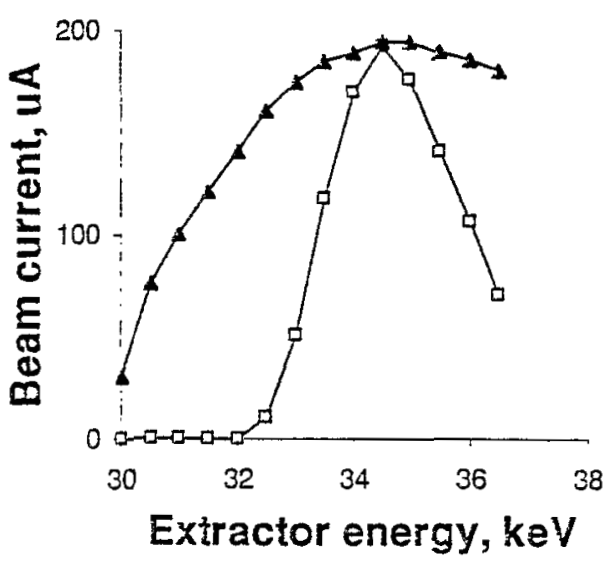

FIGURE 4. Linac transmission vs extractor voltage (acceleration voltage applied to the jet-ionizer cell). Triangles-LEBT optics with the magnetic quadrupole triplet: squares-quad triplets replaced with the decelerating Einzel-lens.

The increase of the ECR current was obtained earlier with an admixture of a few percent of gaseous oxygen to the hydrogen in the source [2]. The oxygen admixture also reduces the molecular $\mathrm{H}_{2}{ }^{+}$ion production i.e. improves the dissociation ratio in the source. The magnetic field shape in the ECR region also affects the dissociation ratio. The optimization of the ECR source parameters gave rise to an increase of the main beam intensity and reduction of the half energy, lower polarization component to below $10 \%$. The ECR operation in a pulsed mode was also studied. A significant molecular component suppression was also observed in a pulsed operation (see Fig.3), due to a difference in the rise time for main and half energy beam components. In a pulsed operation the molecular component is about $5 \%$. As a result the polarization at $200 \mathrm{MeV}$ was increased to $75 \%$. The next step was a suppression of the lower energy component during acceleration to $35 \mathrm{keV}$ after the ionizer and in the LEBT. The twogap acceleration system was upgraded to three-gaps and the voltage in the first gap was tuned to suppress the half energy component. The voltages at the second and third gaps were adjusted to minimize the main component losses. The triplet of magnetic quadrupole lenses at the OPPIS exit was replaced by a single deceleration einzel lens.

The combined effect of these modifications is a significant (almost ten times) suppression of the beam transmission in LEBT for the lower energy molecular origin beam (see Fig.4). As a result of these upgrades, a polarization at $200 \mathrm{MeV}$ of $80-82 \%$ was measured in both p-Carbon and p-Deutron polarimeters.

\section{OPPIS UPGRADE FOR $62 / 3 \mathrm{~Hz}$ REPETITION RATE}

A multi-bunch beam is required for depolarization studies during the AGS energy ramp with a new proton-carbon CNI polarimeter [5]. A four OPPIS pulses will be injected to $A G S$ at $62 / 3 \mathrm{~Hz}$ repetition rate. In 2000 and 2001-02 polarized runs the 
OPPIS was operated at $1 \mathrm{~Hz}$ repetition rate and additional pulses between injection to AGS were directed to $200 \mathrm{MeV}$ polarimeter. A new mode of operation requires a number of upgrades in the source, polarimeter, control and data acquisition system.

The ECR is operated either dc or pulsed, so the higher rate is not a problem. The pulsed high-voltage bias $(32.0 \mathrm{kV})$ of the sodium-jet ionizer cell was a major concern. The ionizer operation at $1 \mathrm{~Hz}$ was reliable, but a quite high (up to $400 \mathrm{~mA}$ ) magnetrontype discharge in the crossed electrical and magnetic fields was observed during the high-voltage pulse. This caused some outgasing, and at higher rate might increase the probability of sparking. An insufficient superconducting solenoid field confinement by the yoke was identified as a problem. A new magnetic field correction coil was attached to the solenoid yoke (see Fig.1), which suppressed almost completely the stray magnetic field in the ionizer high-voltage gap. As a result, the discharge current was reduced at least 10 times and a stable spark-free operation was obtained at $62 / 3$ $\mathrm{Hz}$. At the same time the Sona-transition conditions were also greatly improved due to magnetic field gradient reduction in the zero-crossing region.

The laser cooling system was improved, and stable operation at $62 / 3 \mathrm{~Hz}$ was obtained. While four pulses will be produced in less than $0.5 \mathrm{~s}$, which is followed by about 3 second AGS ramp time, the laser must be pulsed continuously to maintain stable laser power and wavelength operation. The laser service periodicity and lifetime at high rate still have to be studied. For laser circular polarization reversal at $1 \mathrm{~Hz}$ repetition rate, $\lambda / 4$ were plates were inserted in the laser beam by an air-driven slide. Due to large Zeeman shift, the optical pumping can be done by the linear polarized light, which is a combination of both circular polarization components (the wrong component is "sterile" and does not produce any transition, but effective laser power is reduced in half). About 2-3\% polarization losses were obtained experimentally without the $\lambda / 4$ plates at optimal laser tune conditions. It is planned to install a Pockels-cell for laser polarization reversal at high rate.

A Faraday-rotation polarimeter for spin-sequence confirmation now operates routinely at $1 \mathrm{~Hz}$ and will be used for the coming run. A DAQ upgrade for higher repetition rate is in progress for polarization measurements in the source and in a 200 $\mathrm{MeV}$ polarimeter.

\section{REFERENCES}

a. Work supported by the U.S. Department of Energy

1. G. Bunce et al., "Polarized protons at RHIC", Particle World 3. 1 (1092).

2. A. Zelenski et al.. Rev. Sci. Instrum. 73, 888 (2002).

3. A. Zelenski et al., Nucl. Instrum. Methods A402, 185 (1998).

t. A. Zelenski, in Proc. of SPIN 2000. AIP Conf. Proc. 570, 179 (2001).

5. E. Stephenson, privare communications.

6. H. Huang et al., to be published in Proceedings of EP.+C 2002. 